\title{
Insulineffekt im Gehirn reduziert Hungergefühl
}

\author{
Insulin aktiviert bestimmte Hirnregionen und kann so helfen, das Hungergefühl zu regulieren. \\ Darauf deuten neue Untersuchungen von Forscherinnen und Forschern des Deutschen \\ Zentrums für Diabetesforschung (DZD) aus Tübingen hin.
}

\begin{abstract}
Originalie
Stephanie Kullmann, Martin Heni, Ralf Veit, Klaus Scheffler, Jürgen Machann, Hans-Ulrich Häring, Andreas Fritsche, Hubert Preissl. Intranasal insulin enhances brain functional connectivity mediating the relationship between adiposity and subjective feeling of hunger. Scientific Reports | 7: 1627| DOI:10.1038/s41598-01701907-w
\end{abstract}

Das Essverhalten und das Hungergefühl werden von einer Vielzahl an Hormonen reguliert. Eine Schlüsselrolle spielt dabei Insulin, das nicht nur im Körper, sondern auch im Gehirn aktiv ist. Bisher ist bekannt, dass Insulin auf die homöostatische Region im Hypothalamus $^{*}$ wirkt. Es wird allerdings vermutet, dass das Hormon auch in weiteren Hirnregionen aktiv ist. Wissenschaftlern des Instituts für Diabetesforschung und Metabolische Erkrankungen des Helmholtz Zentrum München an der Eberhard-Karls-Universität Tübingen ist es nun gelungen, die Funktion von Insulin im Gehirn und seinen Einfluss auf das subjektive Hungerempfinden weiter zu entschlüsseln.

Um die Wirkungsweise von Insulin besser zu verstehen, haben die Forscherinnen und Forscher gesunden jungen Erwachsenen Insulin zur intranasalen Anwendung gegeben. Durch die Applikation des Hormons über ein Nasenspray wird die Bluthirnschranke umgangen und das Insulin gelangt direkt ins $\mathrm{Ge}$ hirn. In der Studie „schnupften“ 25 gesunde schlanke, zehn übergewichtige und 12 adipöse Erwachsene Insulin oder Placebo. Anschließend wurden die Hirnaktivitäten mit Magnetresonanz-Tomografie erfasst ( $\triangleright$ Abb. 1$)$.

Das Ergebnis: Das intranasale Insulin verbessert bei allen Studienteilnehmern die funktionalen Verbindungen in den präfrontalen Regionen des Ruhestandsnetzwerks (Default Mode Network, DMN ${ }^{* *}$ einer Gruppe von Hirnregionen, die aktiviert werden, wenn der Mensch ruht und keinerlei Aufgaben nachgeht. Diese Region ist zentral für kognitive Prozesse. Darüber hinaus verstärken sich die funktionalen Verbindungen zwischen dem DMN und dem Hippocampus. Diese Veränderungen im Gehirn haben auch einen Einfluss auf das Essverhalten. Sie bewirken, dass sich die Verbindung zwischen Fettleibigkeit und dem Hungergefühl ändert. Eigentlich haben Menschen mit viel

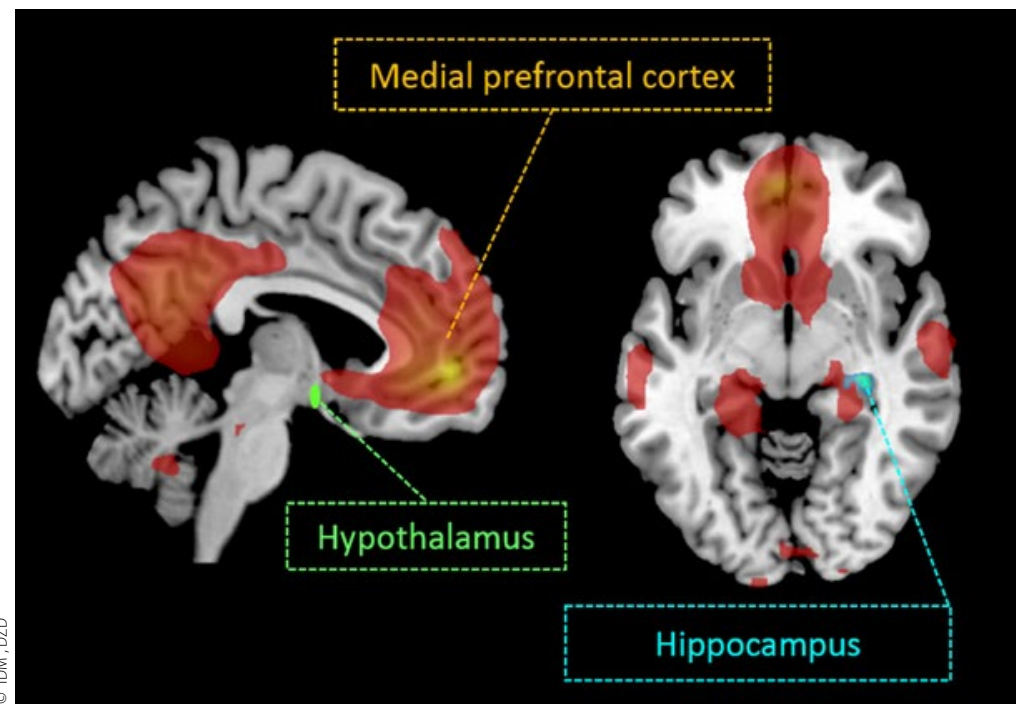

1 Intranasales Insulin steigert die funktionalen Verbindungen in bestimmten kognitiven Hirnregionen (Default Mode Network, DMN) sowie im Hippocampus und Hypothalamus.

viszeralem Fettgewebe ${ }^{* *}$ auch mehr Hunger also jene mit wenig dieses Fettgewebes. Bei einer durch Insulin erhöhten Konnektivität zwischen dem DMN und dem Hippocampus wird diese Verkettung zwischen Fettgewebe und dem subjektiven Hungergefühl unterdrückt. Die betroffenen Teilnehmer hatten nach intranasaler Insulingabe weniger Hunger.

Außerdem beobachteten die Wissenschaftler, dass Insulin im Gehirn auch die Wirkung des Hormons im Körper verbessert. Studienteilnehmer mit einer durch Insulin induzierten erhöhten funktionalen Konnektivität im DMN weisen im Körper eine höhere Insulinempfindlichkeit auf. Das wirkt Adipositas und Typ-2-Diabetes entgegen. Die aktuellen Ergebnisse zeigen, dass Insulin im Gehirn durch eine verbesserte funktionelle Verbindung zwischen kognitiven und homöostatischen Regionen im Gehirn vielleicht helfen kann, das Essenverhalten zu regulieren und abzunehmen.

\footnotetext{
* Der Hypothalamus ist das oberste Regulationszentrum für alle vegetativen und endokrinen Vorgänge. Der Hypothalamus koordiniert Wasser-, Salzhaushalt und Blutdruck. Er sorgt für die Aufrechterhaltung des inneren Milieus (Homöostase) und reguliert die Nahrungsaufnahme.

* Das Default Mode Network DMN (Ruhezustandsnetzwerk) ist eine Gruppe von Gehirnregionen, die aktiv ist, wenn ein Mensch tagträumt, Zukunftspläne macht usw. Es ermöglicht das reizunabhängige Denken.
} 